\title{
Angular-Momentum-Mass Inequality for Axisymmetric Black Holes
}

\author{
Sergio Dain ${ }^{1, *}$ \\ ${ }^{1}$ Albert-Einstein-Institut, am Mühlenberg 1, D-14476, Golm, Germany
}

(Received 21 November 2005; published 16 March 2006)

\begin{abstract}
The inequality $\sqrt{J} \leq m$ is proved for vacuum, asymptotically flat, maximal, and axisymmetric data close to extreme Kerr data. The physical significance of this inequality and its relation to the standard picture of the gravitational collapse are discussed.
\end{abstract}

DOI: 10.1103/PhysRevLett.96.101101

Introduction. - The following conjectures constitute the essence of the current standard picture of the gravitational collapse: (i) Gravitational collapse results in a black hole (weak cosmic censorship), (ii) the spacetime settles down to a stationary final state. If we further assume that at some finite time all the matter fields have fallen into the black hole and hence the exterior region is pure vacuum (for simplicity we discard electromagnetic fields in the exterior), then the black hole uniqueness theorem implies that the final state should be the Kerr black hole. The Kerr black hole is uniquely characterized by its mass $m_{0}$ and angular momentum $J_{0}$. These quantities satisfy the following remarkable inequality

$$
\sqrt{\left|J_{0}\right|} \leq m_{0} .
$$

From Newtonian considerations, we can interpret this inequality as follows [1]: in a collapse the gravitational attraction $\left(\approx m_{0}^{2} / r^{2}\right)$ at the horizon $\left(r \approx m_{0}\right)$ dominates over the centrifugal repulsive forces $\left(\approx J_{0}^{2} / m_{0} r^{3}\right)$.

If the initial conditions for a collapse violate (1) then the extra angular momentum should be radiated away in gravitational waves. However, in an axially symmetric spacetime the angular momentum is a conserved quantity (the Komar integral of the Killing vector, see, for example, [2]). In this case angular momentum cannot be radiated: the angular momentum $J$ of the initial conditions must be equal to the final one $J_{0}$. On the other hand, the mass of the initial conditions $m$ satisfies $m \geq m_{0}$ because gravitational radiation carries positive energy. Then, from inequality (1) we obtain

$$
\sqrt{|J|} \leq m .
$$

More precisely, (i) and (ii) imply that a complete, vacuum, axisymmetric, asymptotically flat data should satisfy inequality (2), where $m$ and $J$ are the mass and angular momentum of the data. Moreover, the equality in (2) should imply that the data are a slice of extreme Kerr spacetime. This is a similar argument to the one used by Penrose [3] to obtain the inequality between mass and the area of the horizon on the initial data. As in the case of Penrose inequality, a counter example of (2) will imply that either (i) or (ii) is not true. Conversely a proof of (2) gives
PACS numbers: 04.70.Bw, 04.20.Dw, 04.20.Ex, 04.20.Fy

indirect evidence of the validity of (i) and (ii), since it is very hard to understand why this highly nontrivial inequality should hold unless (i) and (ii) can be thought of as providing the underlying physical reason behind it (see the discussion in [4]). The main result of this Letter is that (2) is true for data close enough to extreme Kerr data.

Inequality (2) is a property of the spacetime and not only of the data, since both quantities $m$ and $J$ are independent of the slicing. It is in fact a property of axisymmetric, vacuum, black hole spacetimes, because a nonzero $J$ (in vacuum) implies a nontrivial topology on the data and this is expected to signal the presence of a black hole. The physical interpretation of (2) is the following: if we have a stationary vacuum black hole (i.e., Kerr) and add to it axisymmetric gravitational waves, then the spacetime will still have a (nonstationary) black hole; these waves will only increase the mass and not the angular momentum of the spacetime because they are axially symmetric. Since inequality (1) is satisfied for the Kerr black hole we get (2).

The Kerr black hole has been proved to be unique among stationary solutions (see the review articles $[5,6]$ and references therein). There exists also linear stability studies for the Kerr black hole $[7,8]$. The result presented here is the first nonlinear one which proves the relevance of the Kerr black hole among nonstationary solutions of Einstein equations.

The variational principle. - Inequality (2) suggests the following variational principle: The extreme Kerr initial data are the absolute minimum of the mass among all axisymmetric, vacuum, asymptotically flat, and complete initial data with fixed angular momentum. With the extra assumption that the data are maximal, this variational principle can be formulated in a very simple form [9]. A maximal initial data set for Einstein's vacuum equations consists in a Riemannian metric $\tilde{h}_{a b}$, and a trace-free symmetric tensor field $\tilde{K}^{a b}$, such that the vacuum constraint equations

$$
\begin{gathered}
\tilde{D}_{b} \tilde{K}^{a b}=0, \\
\tilde{R}-\tilde{K}_{a b} \tilde{K}^{a b}=0,
\end{gathered}
$$

are satisfied; where $\tilde{D}_{a}$ and $\tilde{R}$ are the Levi-Civita connection and the Ricci scalar associated with $\tilde{h}_{a b}$. In these 
equations the indexes are moved with the metric $\tilde{h}_{a b}$ and its inverse $\tilde{h}^{a b}$.

We assume that the initial data are axially symmetric, that is, there exists an axial Killing vector $\eta^{a}$ such that

$$
\mathcal{L}_{\eta} \tilde{h}_{a b}=0, \quad \mathcal{L}_{\eta} \tilde{K}_{a b}=0,
$$

where $\mathcal{L}$ denotes the Lie derivative. The Killing vector $\eta^{a}$ is assumed to be hypersurface orthogonal on the data. Under these conditions, the metric $\tilde{h}_{a b}$ can be characterized by two functions $q, v$; we specify them using Brill's ansatz [10]. Let $\rho, z, \phi$ be cylindrical coordinates in $\mathbb{R}^{3}$. We write the metric in the form

$$
\tilde{h}_{a b}=e^{v} h_{a b},
$$

where the conformal metric $h_{a b}$ is given by

$$
h=e^{-2 q}\left(d \rho^{2}+d z^{2}\right)+\rho^{2} d \phi^{2} .
$$

In these coordinates we have $\eta^{a}=(\partial / \partial \varphi)^{a}$. The norm of $\eta^{a}$ with respect to the metric $\tilde{h}_{a b}$ will be denoted by $X$

$$
X=\eta^{a} \eta^{b} \tilde{h}_{a b}=e^{v} \rho^{2} .
$$

The function $q$ is assumed to be smooth with respect to the coordinates $\rho, z$. At the axis we impose the regularity condition

$$
q(\rho=0, z)=0 .
$$

This condition implies that the conformal metric $h_{a b}$ is well defined in $\mathbb{R}^{3}$. At infinity we assume the following falloff

$$
q=o\left(r^{-1}\right), \quad q_{, r}=o\left(r^{-2}\right) .
$$

These fall off conditions imply that the mass of the physical metric $\tilde{h}_{a b}$ is contained in the conformal factor $v$. This function is allowed to be singular at some points at the axis, these singularities represent the extra asymptotic ends of the data. In the present case, since we will study small deviation from the extreme Kerr data, we only have one extra end, the corresponding singular point of $v$ will be chosen to be at the origin.

The relevant part of the second fundamental form $\tilde{K}^{a b}$ is characterized by a potential $Y$ which is calculated as follows. Define the vector $\tilde{S}^{a}$ by

$$
\tilde{S}_{a}=\tilde{K}_{a b} \eta^{b}-X^{-1} \tilde{\eta}_{a} \tilde{K}_{b c} \eta^{b} \eta^{c},
$$

where $\tilde{\eta}_{a}=\tilde{h}_{a b} \eta^{b}$. Then, define $K_{a}$ by

$$
K_{a}=\tilde{\epsilon}_{a b c} \tilde{S}^{b} \eta^{c},
$$

where $\tilde{\epsilon}_{a b c}$ is the volume element of $\tilde{h}_{a b}$. Using Eqs. (3) and (5) and the Killing equation we obtain

$$
\tilde{D}_{[b} K_{a]}=0 .
$$

Hence, there exists a scalar function $Y$ such that $K_{a}=$ $\tilde{D}_{a} Y$. The angular momentum $J$ of the data is given by the value of $Y$ at the axis. More precisely, we have

$$
J=\frac{1}{8}[Y(\rho=0,-z)-Y(\rho=0, z)], \quad z \neq 0 .
$$

Summarizing, for any data $\left(\tilde{h}_{a b}, \tilde{K}^{a b}\right)$ which satisfy the assumptions above, we have a pair $(v, Y)$. The function $v$ is calculated from the metric $\tilde{h}_{a b}$, it contains the mass of the data. The potential $Y$ is calculated from $\tilde{K}^{a b}$ and it contains the angular momentum of the data. These functions will be our fundamental variables. For a given $(v, Y)$ we can calculate $q$ from the constrain equation (4) (see the discussion in [9]).

Consider the functional defined in [9]

$$
\mathcal{M}(v, Y)=\frac{1}{32 \pi} \int_{\mathbb{R}^{3}}\left(|\partial v|^{2}+\rho^{-4} e^{-2 v}|\partial Y|^{2}\right) d \mu,
$$

where $d \mu=\rho d z d \rho d \phi$ is the volume element in $\mathbb{R}^{3}$ and $\partial$ denotes partial derivative with respect to $\rho$ and $z$; that is $|\partial v|^{2}=v_{, z}^{2}+v_{, \rho}^{2}$. Note that this functional does not depend on $q$. In [9] it has been proved that the following bound holds for every maximal data

$$
m \geq \mathcal{M} .
$$

Equation (16) allows us to formulate the variational principle mentioned above in terms of the functional $\mathcal{M}$ which depends only on two free functions $(v, Y)$ : we want to prove that the extreme Kerr data are a minimum of $\mathcal{M}$ among all $(v, Y)$ which satisfy the following boundary conditions.

Let $v_{0}$ and $Y_{0}$ denote the extreme Kerr initial data. These are explicit functions (see [11]) which depend on a free parameter $J$, the angular momentum of the data. As it was pointed out above, the function $v_{0}$ is singular at the origin since extreme Kerr data has two asymptotic ends, nevertheless the mass functional (15) is finite and gives the total mass of the extreme Kerr data $\mathcal{M}\left(v_{0}, Y_{0}\right)=\sqrt{|J|}$. Set

$$
v=v_{0}+\alpha, \quad Y=Y_{0}+y .
$$

The functions $(\alpha, y)$ are required to have a falloff compatible with asymptotic flatness. For $y$ we need also to prescribe boundary conditions at the axis in order to impose that the angular momentum of $Y$ is the same as the one of $Y_{0}$. From Eq. (14) we get that $y$ should vanishes at the axis. To simplify the analysis we will further assume that $y$ vanishes in a whole neighborhood of the axis. Note that for $\alpha$ no extra boundary conditions are imposed, we just require that it is a regular function in $\mathbb{R}^{3}$. These considerations are made precise in the following definition of the Banach space $\mathcal{B}$. Let $\Omega$ be a (unbounded) domain in $\mathbb{R}^{3}$. We introduce the weighted spaces of $C^{1}$ functions in $\Omega$

$$
\|f\|_{C_{\beta}^{1}(\Omega)}=\sup _{x \in \Omega}\left\{\sigma^{-\beta}|f|+\sigma^{-\beta+1}|\partial f|\right\},
$$

with $\beta<-1 / 2$ and $\sigma=\sqrt{r^{2}+1}, r=\sqrt{\rho^{2}+z^{2}}$. Let $\rho_{0}>0$ be a constant and $K_{\rho_{0}}$ be the cylinder $\rho \geq \rho_{0}$ in 
$\mathbb{R}^{3}$. We define the domain $\Omega_{\rho_{0}}$ by $\Omega_{\rho_{0}}=\mathbb{R}^{3} \backslash K_{\rho_{0}}$. The perturbation $y$ is assumed to vanish in $K_{\rho_{0}}$. To simplify the notation we will write $\varphi \equiv(\alpha, y)$ and $u_{0} \equiv\left(v_{0}, Y_{0}\right)$. The Banach space $\mathcal{B}$ is defined by

$$
\|\varphi\|_{\mathcal{B}} \equiv\|\alpha\|_{C_{\beta}^{1}\left(\mathbb{R}^{3}\right)}+\|y\|_{C_{\beta}^{1}\left(\Omega_{\rho_{0}}\right)} .
$$

We consider $\mathcal{M}$ as a functional on $\mathcal{M}: \mathcal{B} \rightarrow \mathbb{R}$. Our main result is given by the following theorem proved in [11].

Theorem 1. - The functional $\mathcal{M}: \mathcal{B} \rightarrow \mathbb{R}$ defined by (15) has a strict local minimum at $u_{0}$. That is, there exist $\epsilon>0$ such that

$$
\mathcal{M}\left(u_{0}+\varphi\right)>\mathcal{M}\left(u_{0}\right),
$$

for all $\varphi \in \mathcal{B}$ with $\|\varphi\|_{\mathcal{B}}<\epsilon$ and $\varphi \neq 0$.

Using, essentially, inequality (16), from this theorem we deduce the following corollary.

Corollary 1. - Let $\left(\tilde{h}_{a b}, \tilde{K}^{a b}\right)$ be a maximal, axisymmetric, vacuum, initial data with mass $m$ and angular momentum $J$, such that the metric satisfies (6), (7), (9), and (10). Define $\varphi$ as above. Then, there exists $\epsilon>0$ such that for $\|\varphi\|_{\mathcal{B}}<\epsilon$ the inequality (2) holds. Moreover, $m=\sqrt{J}$ in this neighborhood if and only if the data are the extreme Kerr data.

The main ideas in the proof of Theorem 1 are the following. Consider the real-valued function

$$
i_{\varphi}(t)=\mathcal{M}\left(u_{0}+t \varphi\right)
$$

The first variation of $\mathcal{M}$ is given by $i_{\varphi}^{\prime}$, where prime denotes derivate with respect to $t$. In [9] it has been proved that the extreme Kerr initial data is a critical point of $\mathcal{M}$, that is we have

$$
i_{\varphi}^{\prime}(0)=0, \quad \text { for all } \varphi \in \mathcal{B} .
$$

If we compute the second variation $i_{\varphi}^{\prime \prime}(t)$ it is not obvious that it is positive at the critical point $t=0$. However, using a remarkable identity found by Carter [12], and the equivalence (up to boundary terms) between the functional $\mathcal{M}$ and Carter's Lagrangian (see [9]) it is possible to prove (see [11]) that

$$
i_{\varphi}^{\prime \prime}(0) \geq 0, \quad \text { for all } \varphi \in \mathcal{B} .
$$

Equation (23) can be taken as an interpretation of Carter's identity. This equation is a crucial necessary condition to guarantee that $u_{0}$ is a local minimum, however it is not sufficient. In order to provide a sufficient condition we need to prove that $i_{\varphi}^{\prime \prime}(0)$ is coercive with respect to some appropriate norm. This last step was done in [11] (see Lemma 3.1 in this reference).

This work has been supported by the Sonderforschungsbereich SFB/TR 7 of the Deutsche Forschungsgemeinschaft.

*Electronic address: dain@aei.mpg.de

[1] R. Wald, Phys. Rev. Lett. 26, 1653 (1971).

[2] R. Wald, General Relativity (University of Chicago, Chicago, 1984).

[3] R. Penrose, Riv. Nuovo Cimento 1, 252 (1969).

[4] R. Wald, in Black Holes, Gravitational Radiation and the Universe, edited by B. R. Iyer and B. Bhawal, Fundamental Theories of Physics Vol. 100 (Kluwer Academic, Dordrecht, 1999), p. 69.

[5] B. Carter, in The Eighth Marcel Grossmann Meeting, Part A, B (Jerusalem, 1997) (World Scientific, River Edge, NJ, 1999), p. 136.

[6] P. T. Chruściel, in The Conformal Structure of Spacetimes: Geometry, Analysis, Numerics, edited by J. Frauendiener and H. Friedrich, Lecture Notes in Physics Vol. 604 (Springer, New York, 2002), p. 61.

[7] B. F. Whiting, J. Math. Phys. (N.Y.) 30, 1301 (1989).

[8] H. R. Beyer, Commun. Math. Phys. 221, 659 (2001).

[9] S. Dain, gr-qc/0508061.

[10] D. Brill, Ann. Phys. (N.Y.) 7, 466 (1959).

[11] S. Dain, gr-qc/0511087.

[12] B. Carter, Phys. Rev. Lett. 26, 331 (1971). 